\title{
EFFECT OF NANOFLUIDS AND MASS FLOW RATE OF AIR ON HEAT TRANSFER RATE IN AUTOMOBILE RADIATOR BY CFD ANALYSIS
}

\author{
J.R. Patel ${ }^{1}$, A.M. Mavani ${ }^{2}$ \\ ${ }^{1}$ PG Student (Thermal), Mechanical Engineering Department, LDRP-ITR, Gandhinagar, Gujarat, India \\ ${ }^{2}$ Associate Professor, Mechanical Engineering Department, LDRP-ITR, Gandhinagar, Gujarat, India
}

\begin{abstract}
Air has the largest influence on the overall heat transfer rate of automobile radiator. It is generally known that the velocity of the airflow through the radiator is a function of the vehicle speed and the "heat transferred by a radiator is a function of the airflow rate across the radiator" This paper presents a Computational Fluid Dynamics (CFD) modeling simulation of mass flow rate of air passing across the tubes and coolants in to the tubes of an automotive radiator. An introduction to Nanofluids is termed as the next generation heat transfer elements. The mass concentration of nano particles is proportional to the rate of heat transfer within critical limit. Air flow and coolants simulation is conducted using commercial software STAR CCM+. The CFD process starts with defining the water type geometric clean up and meshing using the Hyper mesh V11.0 software and then it is followed by the meshing which create the surface mesh as well as volume mesh accordingly. After meshing, the boundary conditions are defining before solving that represents flow fields of the simulation. The flow characteristics are then analyzed, compared and verified according to known physical situation and existing experimental data.
\end{abstract}

Keywords: Radiator, Modeling, simulation, Heat transfer, Nanofluids, CFD

$* * *$

\section{INTRODUCTION}

CFD is a science that can be helpful for studying fluid flow, heat transfer, chemical reactions etc by solving mathematical equations with the help of numerical analysis. CFD employs a very simple principle of resolving the entire system in small cells or grids and applying governing equations on these discrete elements to find numerical solutions regarding pressure distribution temperature gradients, flow parameters and the like in a shorter time at a lower cost because of reduced required experimental work. Automobile Radiator are becoming highly power-packed with increasing power to weight or volume ratio. Increased demand on power packed radiators, which can dissipate maximum amount of heat for any given space. Nanofluids give higher heat transfer rate than base fluids. This study involves CFD simulation of the mass flow rate of air and heat transfer of fin and tube heat exchanger (radiator) at various coolants (Nanofluids). In this paper, CFD used to simulate flow and heat transfer in tube and fin heat exchanger. Coolants flow through the tubes of the radiator, heat is transferred through the fins and tube walls to the air by conduction and convection. The radiator of maruti van car is analyzed to get heat transfer rate at different mass flow rate of air and coolants in this study. CFD analysis gives accurate and exact result.

\section{EXPERIMENTAL HEAT TRANSFER CALCULATION}

Radiator is considered as a tube and fin type heat exchanger and Experimental maruti van radiator with dimension are as under.

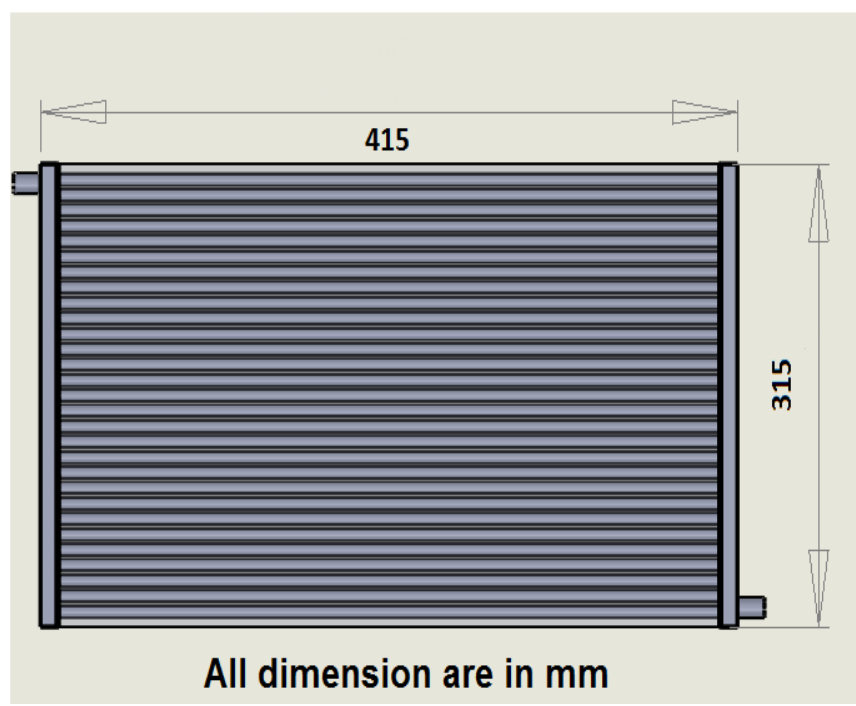

Fig 2.1: Experimental Radiator

Shell (fin) side Data:-

Media:-Air

Temperature: $-30^{\circ} \mathrm{C}$

Inlet Velocity: - $30 \mathrm{Kmph}$ (Vehicle Speed)

Outlet Pressure: - 1.01325 bar 


\section{Tube Side Data:}

Diameter of Tube: $-8 \mathrm{~mm}$

No. of Tubes: -57

Media: - water + Ethanol (50\%), Cuo/water and $\mathrm{Tio}_{2} /$ water

Temperature (Engine):- $107{ }^{\circ} \mathrm{C}$

Mass flow rate of coolants:- $2.5 \mathrm{Kg} / \mathrm{s}$
Outlet Pressure: - 1.01325 bar

Mass flow rate, Overall Heat transfer co-efficient and Heat transfer Rate are calculated as per its respective equations are:

Table 1: Experimental Results of Company

\begin{tabular}{|c|c|c|c|c|c|c|c|}
\hline $\begin{array}{l}\text { Sr. } \\
\text { no. }\end{array}$ & $\begin{array}{l}\text { Velocit } \\
\mathrm{y} \text { of } \\
\mathrm{Car} \\
\mathrm{Km} \text { ph }\end{array}$ & $\begin{array}{l}\text { velocity of Car } \\
\mathrm{m} / \mathrm{s}\end{array}$ & Coolants & $\begin{array}{l}\text { Engine } \\
\text { temperature } \\
\text { inlet Tube } \\
\text { Side }\left({ }^{\circ} \mathrm{C}\right)\end{array}$ & $\begin{array}{l}\text { Tube Side } \\
\text { Outlet } \\
\text { Temperate } \\
\left({ }^{0} \mathrm{C}\right) \text { (Experi } \\
\text { mental) }\end{array}$ & $\begin{array}{l}\text { Fin Side } \\
\text { inlet } \\
\text { temperature } \\
\left({ }^{0} \mathrm{C}\right)\end{array}$ & $\begin{array}{l}\text { Fin Side } \\
\text { Outlet } \\
\text { temperature } \\
\left({ }^{0} \mathrm{C}\right) \text { (Experi } \\
\text { mental) }\end{array}$ \\
\hline 1 & 30 & 8.33333 & \multirow{4}{*}{$\begin{array}{l}\text { water+ } \\
\text { Ethanol }\end{array}$} & 107 & 101.90 & 30 & 120.33 \\
\hline 2 & 50 & 13.88889 & & 107 & 93.95 & 30 & 111.53 \\
\hline 3 & 80 & 22.22222 & & 107 & 77.85 & 30 & 87.55 \\
\hline 4 & 100 & 27.77778 & & 107 & 76.33 & 30 & 89.23 \\
\hline 5 & 30 & 8.33333 & \multirow{4}{*}{$\begin{array}{l}\mathrm{CuO} / \text { wat } \\
\text { er }\end{array}$} & 107 & 85.12 & 30 & 88.45 \\
\hline 6 & 50 & 13.88889 & & 107 & 79.88 & 30 & 86.53 \\
\hline 7 & 80 & 22.22222 & & 107 & 76.53 & 30 & 87.65 \\
\hline 8 & 100 & 27.77778 & & 107 & 74.93 & 30 & 91.50 \\
\hline 9 & 30 & 8.33333 & \multirow{4}{*}{$\begin{array}{l}\mathrm{Tio}_{2} / \text { wat } \\
\text { er }\end{array}$} & 107 & 86.25 & 30 & 88.33 \\
\hline 10 & 50 & 13.88889 & & 107 & 81.53 & 30 & 85.89 \\
\hline 11 & 80 & 22.22222 & & 107 & 77.10 & 30 & 87.55 \\
\hline 12 & 100 & 27.77778 & & 107 & 75.33 & 30 & 90.73 \\
\hline
\end{tabular}

Mass flow rate $\mathrm{m}=\mathrm{A} * \mathrm{~V} *$ \&, Overall Heat transfer Coefficient $1 / \mathrm{U}=1 / \mathrm{h}_{\mathrm{i}}+1 / \mathrm{h}_{\mathrm{o}}$, Heat transfer area $\mathrm{A}=\mathrm{n} * \prod * \mathrm{~d} * \mathrm{~L}$ $+\left(\mathrm{n} 1 * 11 * \mathrm{~b} 1-\mathrm{n} * \prod * \mathrm{~d}\right), L M T D \quad \theta m=\theta 1-\theta 2 / \ln (\theta 1)$ $\theta 2$ ) and Heat transfer rate $Q=U A \theta m$
Total heat transfer rate of Cuo/water at $1.2 \mathrm{Kg} / \mathrm{s}$ mass flow rate of Air $Q=U A \theta m$

$=2535 * 0.989942 * 33.87$

$Q=8.499 * 10^{4} \mathrm{KW}$

Table 2: Summary of Experimental Results

\begin{tabular}{|c|c|c|c|c|c|c|c|}
\hline Sr. no. & $\begin{array}{l}\text { Engine } \\
\text { temperature } \\
\text { inlet Tube } \\
\text { Side }\left({ }^{0} \mathrm{C}\right)\end{array}$ & velocity of Car m/s & Coolants & $\begin{array}{l}\text { Tube Side } \\
\text { Outlet } \\
\text { Temperate } \\
\left({ }^{0} \mathrm{C}\right) \text { (Experime } \\
\text { ntal) }\end{array}$ & $\begin{array}{l}\text { Fin Side Outlet } \\
\text { temperature } \\
\left({ }^{0} \mathrm{C}\right) \text { (Experime } \\
\text { ntal) }\end{array}$ & $\begin{array}{l}\text { Mass } \\
\text { flow } \\
\text { rate of } \\
\text { air } \\
\mathrm{Kg} / \mathrm{s}\end{array}$ & $\begin{array}{l}\text { Heat transfer } \\
\text { rate }(\mathrm{KW})\end{array}$ \\
\hline 1 & 107 & 8.33333 & \multirow{4}{*}{$\begin{array}{l}\text { water+ } \\
\text { Ethanol }\end{array}$} & 101.90 & 120.33 & 1.2 & 90.12 \\
\hline 2 & 107 & 13.88889 & & 93.25 & 111.53 & 2 & 92.20 \\
\hline 3 & 107 & 22.22222 & & 77.85 & 87.55 & 3 & $1.91 * 10^{5}$ \\
\hline 4 & 107 & 27.77778 & & 76.33 & 89.23 & 4 & $2.18 * 10^{5}$ \\
\hline 5 & 107 & 8.33333 & \multirow{4}{*}{$\begin{array}{l}\mathrm{CuO} / \\
\text { water }\end{array}$} & 85.12 & 88.45 & 1.2 & $8.49 * 10^{4}$ \\
\hline 6 & 107 & 13.88889 & & 79.88 & 86.53 & 2 & $1.33 * 10^{5}$ \\
\hline 7 & 107 & 22.22222 & & 76.53 & 87.65 & 3 & $1.985 * 10^{5}$ \\
\hline 8 & 107 & 27.77778 & & 74.33 & 91.50 & 4 & $2.386 * 10^{5}$ \\
\hline 9 & 107 & 8.33333 & \multirow{4}{*}{$\mathrm{Tio}_{2} /$ water } & 86.25 & 88.33 & 1.2 & $8.398 * 10^{4}$ \\
\hline 10 & 107 & 13.88889 & & 81.53 & 85.89 & 2 & $1.277 * 10^{5}$ \\
\hline 11 & 107 & 22.22222 & & 77.10 & 87.55 & 3 & $1.93 * 10^{5}$ \\
\hline 12 & 107 & 27.77778 & & 75.33 & 90.73 & 4 & $2.26 * 10^{5}$ \\
\hline
\end{tabular}




\section{CFD ANALYSIS}

\subsection{Modeling of Radiator}

We perform first solid modeling of automobile radiator in Cre $\mathrm{O}$ software and then this solid model is transferred to Hypermesh_64 for water type geometric clean up and this geometric model is transferred to STAR CCM+ for meshing and CFD Analysis.

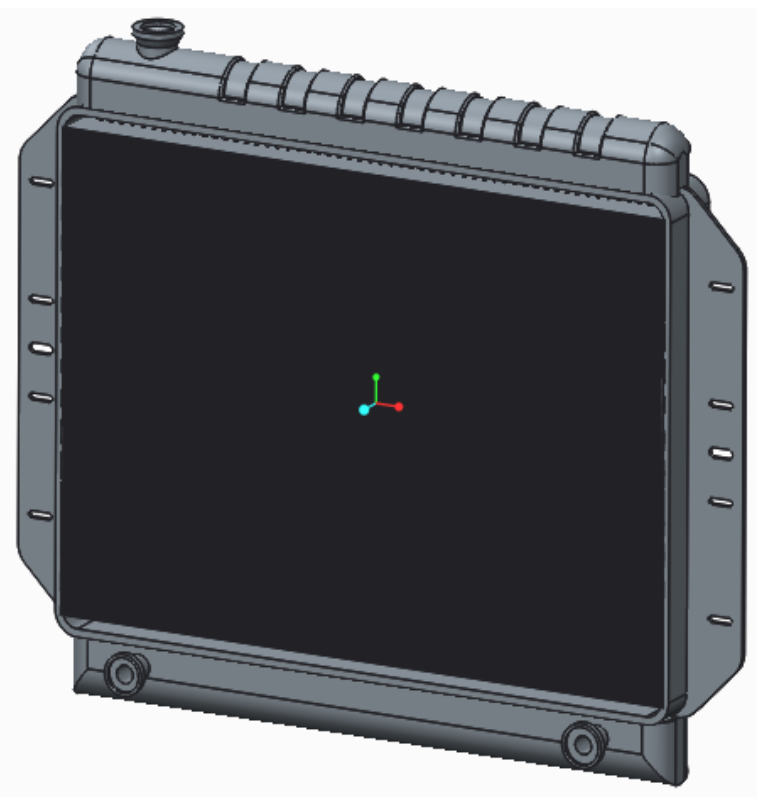

Fig.3.1.1: 3 D Model of Maruti Van Radiator

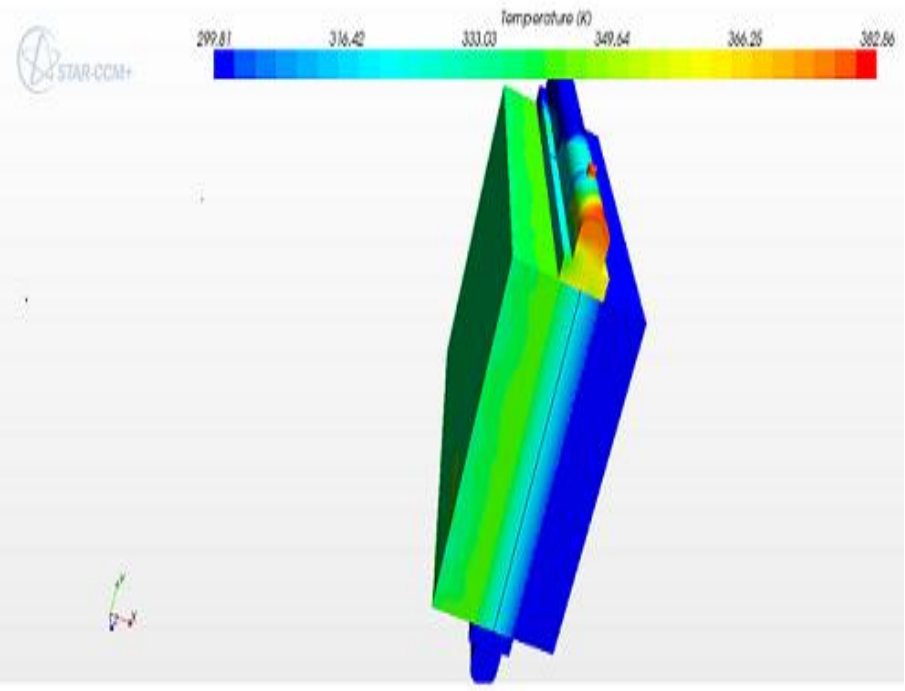

Fig.3.1.2: Water type geometric clean up model of radiator

\subsection{CFD Analysis of Radiator in Star CCM+}

In STAR CCM+, First of all selecting fluid physical model then creates and assign physical continuum. Specify porosity coefficients and then create actual heat exchanger interface. Then specify heat exchanger data specification via UAG table. Create reports, monitors and plots and running the simulation and visualising the results. Using different mass flow rate of air and different coolants as a base fluid and nanofluids, CFD analysis performed.

\subsubsection{Result Analysis}

Case-1: Mass flow rate of air $1.2 \mathrm{Kg} / \mathrm{s}$ and three coolants (base fluid and nanofluids)

Shell (fin) Side Data:-

Media:-Air

Temperature: $-30^{\circ} \mathrm{C}$

Inlet Velocity: - $30 \mathrm{Kmph}$ (Vehicle Speed)

Flow: Turbulent

\section{Tube Side Data:}

Diameter of Tube: $-8 \mathrm{~mm}$

No. of Tubes: -57

Media: - water + Ethanol (50\%), Cuo/water (10\%/90\%) and $\mathrm{Tio}_{2} /$ water $(10 \% / 90 \%)$

Temperature (Engine):- $107{ }^{\circ} \mathrm{C}$

Mass flow rate of coolants:-2.5 Kg/s

Flow: Turbulent

Tube Side Results:

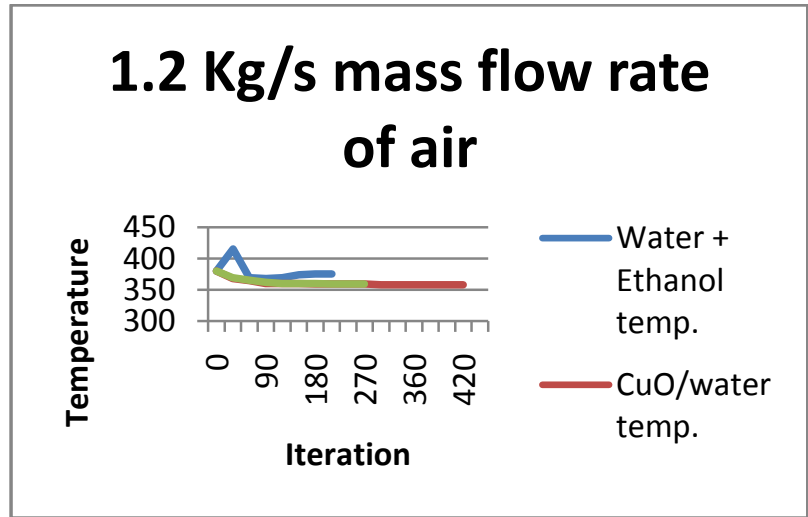

Fig: 3.2.1.1. Coolants Temperature Comparision Graph Shell side results

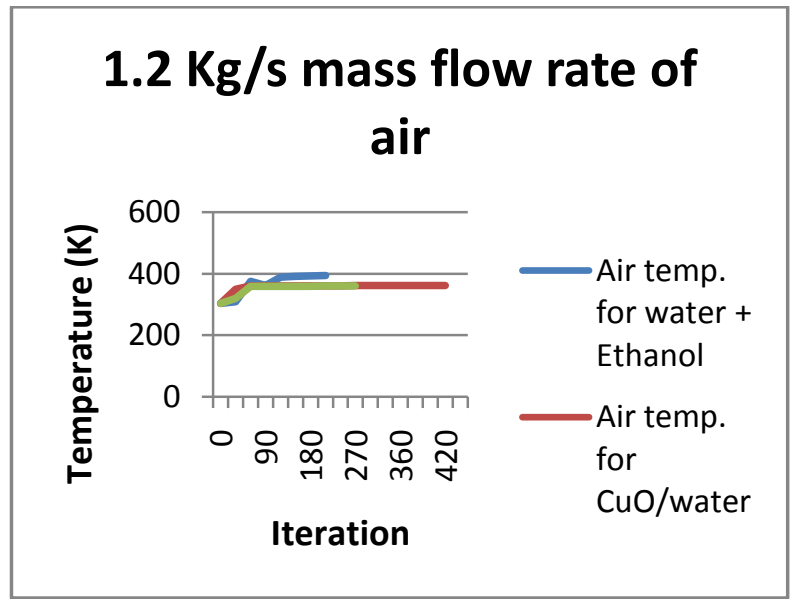

Fig: 3.2.1.2. Air Temperature comparision Graph 


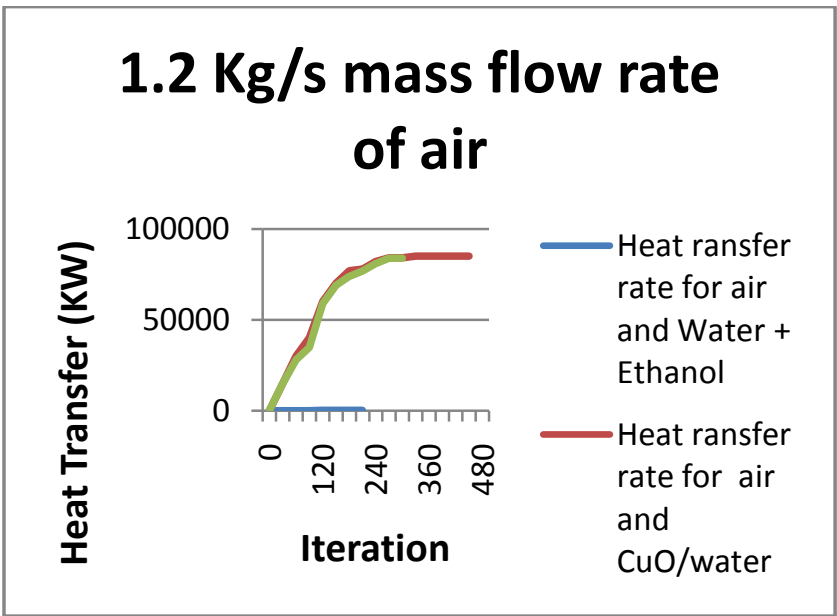

Fig: 3.2.1.2. Heat Transfer Comparision Graph

Case-2: Mass flow rate of air $4 \mathrm{Kg} / \mathrm{s}$ and three coolants (base fluid and nanofluids)

Shell (fin) side Data:-

Media:-Air

Temperature: $-30^{\circ} \mathrm{C}$

Inlet Velocity: - $100 \mathrm{Kmph}$ (Vehicle Speed)

Flow: Turbulent

\section{Tube Side Data:}

Diameter of Tube: $-8 \mathrm{~mm}$

No. of Tubes: - 57

Media: - water + Ethanol (50\%), $\mathrm{CuO} /$ water (10\%/90\%) and $\mathrm{Tio}_{2} /$ water $(10 \% / 90 \%)$

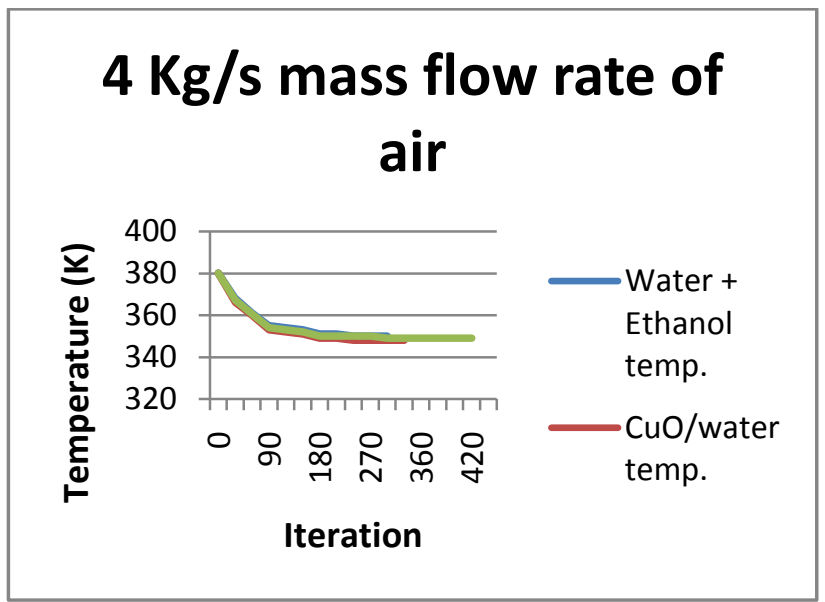

Fig: 3.2.1.4. Coolants Temperature Comparision Graph

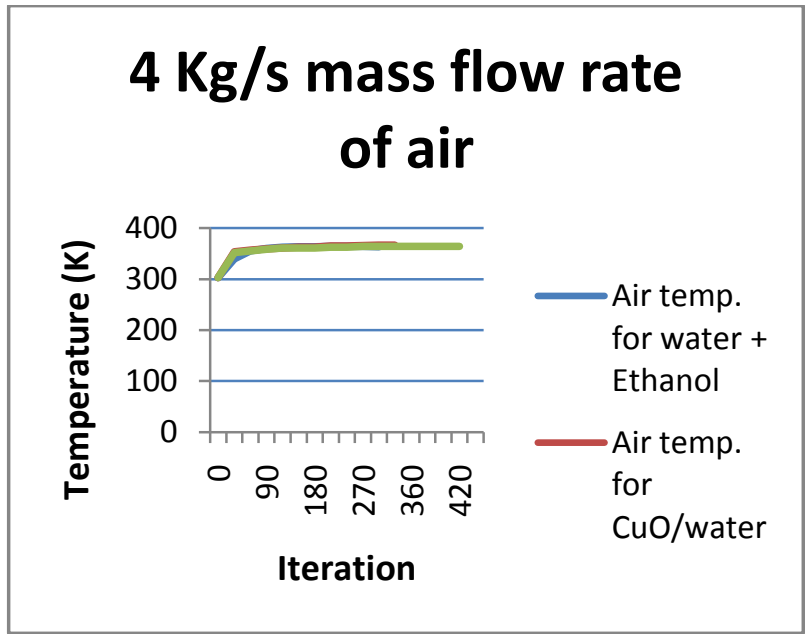

Fig: 3.2.1.5. Air Temperature comparision Graph

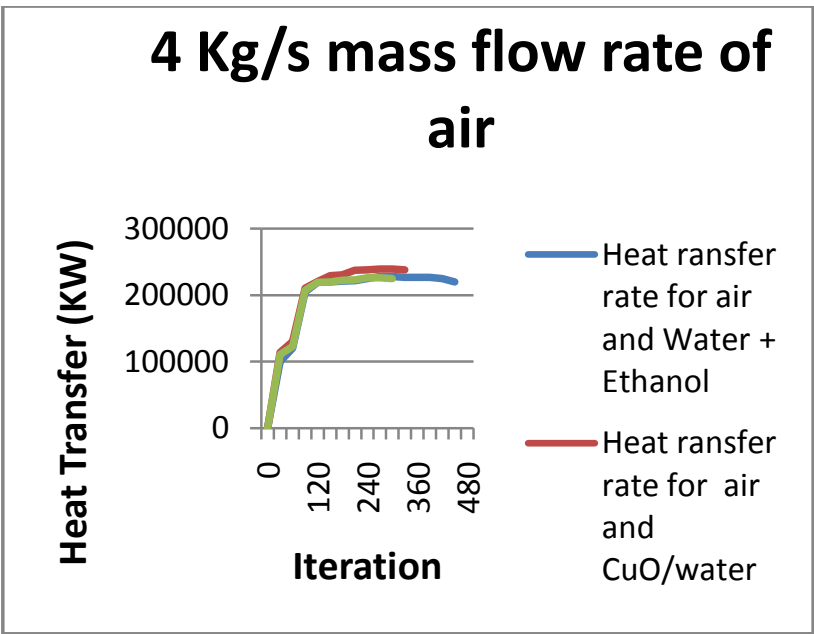

Fig: 3.2.1.6. Heat Transfer Comparision Graph

From above STAR CCM+ analysis there is a $380 \mathrm{~K}$ inlet temperatures of water+ ethanol, $\mathrm{CuO} /$ water and $\mathrm{Tio}_{2} /$ water decreased up to $375 \mathrm{~K}, 357 \mathrm{~K}$ and $359 \mathrm{~K}$ respectively and heat transfer rate higher in nanofluids than base fluids at 1.2 mass flow rate of air. So finally lower coolants outlet temperature and higher heat transfer occurs for $\mathrm{CuO}$ /water nanofluid at $4 \mathrm{Kg} / \mathrm{s}$ mass flow rate of air. 
Table 3: CFD result summary

\begin{tabular}{|c|c|c|c|c|c|c|c|c|}
\hline $\begin{array}{l}\text { Sr. } \\
\text { no. }\end{array}$ & $\begin{array}{l}\text { Veloc } \\
\text { ity of } \\
\text { Car } \\
\mathrm{Km} \\
\text { ph }\end{array}$ & $\begin{array}{l}\text { Mass } \\
\text { flow } \\
\text { rate of } \\
\text { air } \\
\mathrm{Kg} / \mathrm{s}\end{array}$ & $\begin{array}{l}\text { Coolan } \\
\text { ts }\end{array}$ & $\begin{array}{l}\text { Engine } \\
\text { temperatur } \\
\text { e inlet } \\
\text { Tube Side } \\
\left({ }^{0} \mathrm{C}\right) \\
\end{array}$ & $\begin{array}{l}\text { Tube Side } \\
\text { Outlet } \\
\text { Temperate } \\
\left({ }^{0} \mathrm{C}\right)\end{array}$ & $\begin{array}{l}\text { Fin Side } \\
\text { Outlet } \\
\text { temperatur } \\
\text { e }\left({ }^{0} \mathrm{C}\right)\end{array}$ & $\begin{array}{l}\text { Heat transfer } \\
\text { rate }(\mathrm{KW})\end{array}$ & \\
\hline 1 & 30 & 1.2 & \multirow{4}{*}{$\begin{array}{l}\text { water+ } \\
\text { Ethanol }\end{array}$} & 107 & 102 & 121 & 90 & 120.33 \\
\hline 2 & 50 & 2 & & 107 & 94 & 113 & 92 & 111.53 \\
\hline 3 & 80 & 3 & & 107 & 77 & 88 & $1.90 * 10^{5}$ & 87.55 \\
\hline 4 & 100 & 4 & & 107 & 76.5 & 91 & $2.2 * 10^{5}$ & 89.23 \\
\hline 5 & 30 & 1.2 & \multirow{4}{*}{$\begin{array}{l}\mathrm{CuO} / \mathrm{w} \\
\text { ater }\end{array}$} & 107 & 84 & 90 & $8.5 * 10^{4}$ & 88.45 \\
\hline 6 & 50 & 2 & & 107 & 79 & 86 & $1.32 * 10^{5}$ & 86.53 \\
\hline 7 & 80 & 3 & & 107 & 76 & 87 & $1.98 * 10^{5}$ & 87.65 \\
\hline 8 & 100 & 4 & & 107 & 74 & 92 & $2.38 * 10^{5}$ & 91.50 \\
\hline 9 & 30 & 1.2 & \multirow{4}{*}{$\begin{array}{l}\mathrm{Tio}_{2} / \mathrm{wa} \\
\text { ter }\end{array}$} & 107 & 87 & 89 & $8.4 * 10^{4}$ & 86.25 \\
\hline 10 & 50 & 2 & & 107 & 81 & 85 & $1.28 * 10^{5}$ & 81.10 \\
\hline 11 & 80 & 3 & & 107 & 76 & 87 & $1.92 * 10^{5}$ & 76.95 \\
\hline 12 & 100 & 4 & & 107 & 75 & 91 & $2.25 * 10^{5}$ & 76.25 \\
\hline
\end{tabular}

\subsection{CFD Validation}

To validate the CFD results, there is a comparison between CFD result and experimental data which is given below by table:

Table 4: Comparison between Experimental data and CFD results

\begin{tabular}{|c|c|c|c|c|c|c|c|c|}
\hline Sr. no. & Coolants & $\begin{array}{l}\text { Velocity of } \\
\text { Car Km ph }\end{array}$ & $\begin{array}{c}\text { Engine } \\
\text { outlet } \\
\text { temperature } \\
\text { Tube Side } \\
\left({ }^{0} \mathrm{C}\right) \\
\text { (Experiment } \\
\text { al }\end{array}$ & $\begin{array}{c}\text { Engine } \\
\text { outlet } \\
\text { temperatur } \\
\text { e Tube } \\
\text { Side }\left({ }^{0} \mathrm{C}\right) \\
\text { by CFD }\end{array}$ & $\begin{array}{l}\text { Fin Side } \\
\text { Outlet } \\
\text { temperat } \\
\text { ure }\left({ }^{0} \mathrm{C}\right) \\
\text { (Experim } \\
\text { ental) }\end{array}$ & $\begin{array}{c}\text { Fin Side } \\
\text { Outlet } \\
\text { temperatur } \\
\text { e }\left({ }^{0} \mathrm{C}\right) \text { by } \\
\text { CFD }\end{array}$ & $\begin{array}{c}\text { Percentage } \\
\text { of variation } \\
\text { in tube side } \\
\text { temp. }\end{array}$ & $\begin{array}{l}\text { Percent } \\
\text { age of } \\
\text { variati } \\
\text { on in } \\
\text { fin side } \\
\text { temp. }\end{array}$ \\
\hline 1 & \multirow{4}{*}{$\begin{array}{l}\text { water+ } \\
\text { Ethanol }\end{array}$} & 30 & 101.90 & 102 & 120.33 & 121 & 0.10 & 0.33 \\
\hline 2 & & 50 & 93.25 & 94 & 111.53 & 113 & 0.75 & 1.47 \\
\hline 3 & & 80 & 77.85 & 77 & 87.55 & 88 & 0.85 & 0.45 \\
\hline 4 & & 100 & 76.33 & 76.5 & 89.23 & 91 & 0.15 & 0.77 \\
\hline 5 & \multirow{4}{*}{$\begin{array}{c}\mathrm{CuO} / \text { wat } \\
\text { er }\end{array}$} & 30 & 85.12 & 84 & 88.45 & 90 & 1.12 & 0.55 \\
\hline 6 & & 50 & 79.88 & 79 & 86.53 & 86 & 0.12 & 0.53 \\
\hline 7 & & 80 & 76.53 & 76 & 87.65 & 87 & 0.53 & 0.65 \\
\hline 8 & & 100 & 74.33 & 74 & 91.50 & 92 & 0.33 & 0.50 \\
\hline 9 & \multirow{4}{*}{$\begin{array}{c}\mathrm{TiO}_{2} / \text { wate } \\
\text { r }\end{array}$} & 30 & 86.25 & 87 & 88.33 & 89 & 0.75 & 0.67 \\
\hline 10 & & 50 & 81.53 & 81 & 85.89 & 85 & 0.53 & 0.89 \\
\hline 11 & & 80 & 77.10 & 76 & 87.55 & 87 & 1.10 & 0.55 \\
\hline 12 & & 100 & 75.33 & 75 & 90.73 & 91 & 0.33 & 0.27 \\
\hline
\end{tabular}

\section{CONCLUSIONS}

The fluid flow and heat transfer analysis of an automobile radiator is successfully carried out by CFD analysis using STAR CCM+ software. So using $\mathrm{CuO}$ /water nanofluid as a coolant and $4 \mathrm{Kg} / \mathrm{s}$ mass flow rate of air optimum performance of radiator can be performed. CFD Analysis results fairly matches with the experimental results which show that CFD analysis is a good tool for avoiding costly and time consuming experimental work. 


\section{REFERENCES}

[1]. M.Naraki, S.M. Peyghambarzadeh, S.H. Hashemabadi, Y. Vermahmoudi, "Parametric study of overall heat transfer coefficient of $\mathrm{CuO} /$ water nanofluids in a car radiator". International Journal of Thermal Sciences 66 (2013) 82-90

[2]. Creo 2.0 and STAR CCM+ software user guidelines.

[3]. Navid Bozorgan et.al. "Numerical Study on Application of $\mathrm{CuO}-W a t e r$ Nanofluid in Automotive Diesel Engine Radiator" Modern Mechanical Engineering, 2012, 2, 130136

[4]. B Vishnuprasad et.al. "Performance Analysis of Overall heat transfer coefficient using Nanofluids on an Automobile Engine Test Ring" International journal of Mechanical Engineering and robotics research ISSN 2278 - 0149, Vol. 2, No. 4, October 2013

[5] .A.Witry M.H. Al-Hajeri and Ali A. Bondac, 2003, "CFD analysis of fluid flow and heat transfer in patterned roll bonded aluminium radiator", $3^{\text {rd }}$ International conference on CFD, CSIRO, Melbourne, Australia, pp. 1219.

[6]. S.N Sridhara, S.R. Shankapal and V Umesh Babu, "CFD analysis of Fluid Flow \& Heat Transfer in a Single Tube-Fin Arrangement of an Automotive Radiator" International Conference on Mechanical Engineering 2005, Dhaka, Bangladesh, Conference Paper, 2005

[7]. Mr. Krys Bangert et al, "Radiator heat transfer augmentation by changes to wall surface roughness and emmisivity, The University of Sheffield, June-August 2010. [8]. Chidanand K Mangrulkar et.al. "Nanofluid Heat Transfer-A Review" International Journal of Engineering and Technology Volume 3 No. 2, February, 2013 